\title{
ADAPTIVE REAL-TIME VBR VIDEO TRAFFIC PREDICTOR FOR IEEE 802.15.3 WIRELESS AD HOC NETWORKS
}

\author{
Yi-Hsien Tseng, ${ }^{1}$ Eric Hsiao-Kuang Wu, ${ }^{2}$ and Gen-Hucy Chen ${ }^{1}$ \\ ${ }^{1}$ Department of Computer Science and Information Engineering, \\ National Taiwan University, Taipei, TAIWAN \\ ${ }^{2}$ Department of Computer Science and Information Engineering, \\ National Central University, Chung-Li, TAIWAN
}

\begin{abstract}
This paper proposes a new real-time video traffic predictor to meet increasing consumer demand for a high speed high performance wireless broadband network. It analyzes the behaviors and the problems of previous adaptive LMS-type predictors using fixed step size in detail and then proposes an adaptive predictor using variable step size for predicting bandwidth requirement of real-time VBR videos. The proposed adaptive predictor has better ability for handling scene changes and needs not change its parameters for different VBR videos. The simulation shows that the performance of the proposed adaptive predictor is better or near the optimal performance of previous adaptive LMS-type predictors using fixed step size.
\end{abstract}

Keywords: Dynamic bandwidth allocation, traffic prediction, adaptive LMS algorithm

\section{Introduction}

The IEEE 802.15.3[1] standard is designed to connect about 200 wireless devices and the existing standard $2.4 \mathrm{GHz}$ PHY is up to maximum data rate of 55M bits/sec. Moreover, the IEEE 802.15 Task Group 3a (IEEE 802.15.3a) is underway to standardize an UWB [6] PHY with a range of data rates between 110 and $480 \mathrm{M}$ bits/sec. The new UWB PHY of the IEEE 802.15 .3 is ideal for allowing multiple wireless devices to exchange multimedia traffics such as video, audio and digital images.

In addition to high data rates, the IEEE 802.15.3 standard also includes all functionalities needed for reliable QoS. The IEEE 802.15.3 MAC protocol uses TDMA to allocate channel time among devices in order to prevent conflicts and only provides new channel time allocations for a connection if enough bandwidth is available. The elementary topological unit for the IEEE 802.15.3 MAC layer is a piconet, which is a wireless ad hoc data communi- 
cations system in essence. There are a number of independent data devices (DEVs) contained in a piconet that are allowed to exchange frames directly with each other.

The master/slave relationship was adopted for these DEVs; a particular $\mathrm{DEV}$, named piconet coordinator (PNC), acts as the master and the others are slaves. The PNC is also responsible for admission control and channel time allocation. Timing for a piconet is realized by superframes whose three parts are Beacon, CAP and CTAP. When a DEV intends to transmit data, it has to send a request message to the PNC first. The PNC then decides whether the request can be accepted or not according to the available channel time in the superframe. If accepted, the PNC will allocate enough channel time for the $\mathrm{DEV}$ and announce this allocation in the next beacon.

Because the bandwidth in the IEEE 802.15.3 WPANs can be allocated on demand, dynamic bandwidth allocation during the lifetime of a connection should be considered, especially for variable bit rate (VBR) video connection. Since the peak-to-mean ratio of a VBR video is usually high, constant bit rate (CBR) channel allocation which satisfies peak rate requirement often leads to low channel utilization. In contrast, if the channel allocation is not served at a rate close to peak rate, large delays, large queues and packet losses will occur. Therefore, it is important that DEVs should have ability to predict the bandwidth requirement of future superframes in order to support real-time VBR videos.

A dynamic allocation scheme with a novel VBR video traffic predictor is necessary in order to utilize channel efficiently and guarantee the QoS requirement of real-time VBR videos at the same time. By allocating bandwidth equal to the predicted value, only the errors of prediction need to be buffered. Thus, higher channel utilization, small buffers and small delays can be achieved if the prediction is accurate enough.

Several papers which deal with multimedia traffic prediction had been proposed. We simply classify these works into two categories. The first one is model-type prediction [10], [16], [17] which deals with the development of stochastic source models and adopts these models to predict. These models has the ability to capture both the short-range dependent (SRD) and long-range dependent (LRD) [15]. Since VBR videos have been shown that it has a selfsimilar characteristic [9], these stochastic source models can be used for accurate prediction if the parameters of the models were estimated correctly.

Another class of multimedia traffic prediction is adaptive least mean square (LMS) type prediction [2], [5], [12], [14].The adaptive LMS algorithm is well known for its simplicity. Moreover, the adaptive LMS-type prediction neither does require prior knowledge of the video statistics nor does assume stationary. Therefore, they are fit for on-line VBR video prediction for the IEEE 802.15.3 devices. 
However, adaptive LMS-type predictions using fixed step size [13] perform bad performance while scene changes occur. This paper analyzed the effects of scene change on the prediction errors in the adaptive LMS-type prediction which adopts fixed step size. Moreover, this paper proposes an adaptive VBR video predictor based on a variable step-size LMS algorithm [11] in order to overcome the problem of scene change.

The proposed predictor performs much better performance while scene change occurs. Furthermore, instead of the fixed step-size adaptive LMS-type predictor which is hard to determine the optimal parameters for different VBR video traffics in advance, the proposed adaptive predictor adjusts its step size automatically according to the statistics of different VBR video traffics. And the computational complexity of the proposed predictor is also low. By above reasons, the proposed predictor not only satisfies the low-cost requirement of the IEEE 802.15.3 devices but also produces good performance for predicting VBR videos.

The rest of this paper is organized as follows. Related work is introduced in next section. The proposed adaptive VBR video predictor is addressed in Section 3. Analysis and simulation results are shown in Section 4. Finally, this paper concludes with some remarks in Section 5.

\section{Related Work}

There were several papers which deal with traffic prediction proposed in recent years. We simply classify these works into two categories: statistical model-type prediction and adaptive LMS-type prediction. For the first category, the fractional autoregressive integrated moving average (F-ARIMA) [7] model is the most popular one. The F-ARIMA is a self-similar [15] model which has the ability to capture both the SRD and LRD characteristics. It also has been shown that VBR video traffic has a self-similar characteristic [9]. Therefore, the F-ARIMA model is useful for a VBR video traffic predictor.

All parameters of F-ARIMA model should be estimated from the historical traffic data before starting to predict. This causes the bottleneck of computation, especially for the low-cost IEEE 802.15.3 devices. Furthermore, the performance of model-type prediction depends on the parameters of the model can be estimated accurately. It requires large traffic data to estimate the parameters precisely. Therefore, the model based prediction does not suit for bandwidth prediction for real-time VBR videos.

The second category is the adaptive LMS-type prediction. The adaptive LMS algorithm has been wide used in many domains due to its simplicity and relatively good performance. Moreover, the adaptive LMS-type prediction does not require any prior knowledge of the video statistics and does not assume video contents to be stationary. Thus, it well suits for bandwidth pre- 
diction for on-line real time VBR videos which can be non-stationary. A pth order $k$-step linear predictor has the form as:

$$
\widehat{x}(n+k)=\sum_{i=0}^{p-1} w_{n}(l) x(n-l)=W_{n}^{T} X(n)
$$

The $k$-step linear predictor estimates $x(n+k)$ using a linear combination of the current and previous values of $X(n)$. In an adaptive LMS algorithm, $W_{n}$ is the time varying coefficient vector obtained by minimizing the mean square error. The initial vector $W_{0}$ can be assigned any value and $W_{n}$ is updated using the recursive equation as:

$$
W_{n+1}=W_{n}+\mu e(n) X(n)
$$

Since it is difficult to select the proper value of $\mu$ to guarantee convergence, the normalized LMS (NLMS) is often used in practice. The NLMS is a modification of LMS algorithm where the update equation is changed as:

$$
W_{n+1}=W_{n}+\frac{\mu e(n) X(n)}{\|X(n)\|^{2}}
$$

The NLMS will converge in the mean [13] if $0<\mu<2$. Large $\mu$ causes a faster convergence and quicker response to statistic change. However, after convergence, misadjustment is larger. In contrast, the use of a small $\mu$ results in a slower convergence with smaller misadjustment. It is a tradeoff.

[2] proposed a dynamic bandwidth allocation using the NLMS predictor with the fixed value of $\mu$ to support real-time VBR video under RCBR network service model. [5] adopted the fixed step-size NLMS predictor for traffic management of ATM networks. [12] proposed method based on a scene change identification to improve the forecasting performance. They made $X(n)$ to forget historical data while scene changes occur and still used fixed step size.

[14] proposed a scene change indicator for real-time VBR MPEG videos and dynamically controlled the step size $\mu$ between two values: $\mu_{\text {default }}$ and STEP_JUMP $\times \mu_{\text {default }}$. STEP_JUMP is a constant. A scene change is recognized by indicator as:

$$
\frac{|e(n)|}{\sum_{i=n-k}^{n} x(i) l(k+1)}>\text { Threshold }
$$

The step size $\mu$ is increased to STEP_JUMP times $\mu_{\text {default }}$ when a scene change is detected. In contrast, the step size is returned to the initial default value if the normalized prediction error is less than or equal to the threshold value.

Unlike [14] whose step size of $\mu$ is determined by indicator using experienced values of STEP_JUMP, Threshold and the default value $\mu_{\text {default }}$, this 
paper adopts a variable step size LMS algorithm [11] to control the step size for handling scene changes and needs not to determine the value of $\mu$ in advance. The performance of the proposed predictor is better than the other LMS-type predictors which use fixed step size.

\section{Proposed NLMS Adaptive Predictor}

This paper proposes an adaptive predictor based on the variable step size LMS (VSSLMS) algorithm proposed in [11]. This section introduces to the VSSLMS algorithm first and then describes our modification to the VSSLMS algorithm in order to obtain better performance for predicting different VBR videos.

The VSSLMS algorithm adjusts the step size by the square of the prediction error. The motivation of the VSSLMS algorithm is that a large prediction error will cause the step size to increase to provide faster tracking, whereas a small prediction error will result in a decrease in the step size to obtain smaller misadjustment.

The VSSLMS algorithm is the same as the LMS algorithm except the step size of the VSSLMS is updated dynamically. The step-size update equation is expressed as:

$$
\mu_{n+1}=\alpha \mu_{n}+\gamma e^{2}(n)
$$

where $0<\alpha<1, \gamma>0$, and $\mu_{n+1}$ is set to $\mu_{\max }$ or $\mu_{\min }$ when it falls above or below these upper bound and lower bound, respectively. The constant $\mu_{\max }$ should be chosen to ensure that the MSE of the VSSLMS algorithm remains bounded and is normally selected near the point of instability of fixed step-size LMS algorithm to provide the maximum possible convergence. The constant $\mu_{\min }$ is chosen as a compromise between the desired level of steadystate misadjustment and the required tracking capability. Usually, the value of $\mu_{\text {min }}$ is near the one of $\mu$ that would be chosen for the fixed step-size algorithm.

The parameter $\alpha$ is selected from the range $(0,1)$ in order to provide exponential forgetting. The parameter $\gamma$ controls the convergence time and the level of misadjustment. The value of $\gamma$ is usually small. The additional computational overhead of the VSSLMS algorithm is one update for $\mu$ value at each iteration, so that the computational complexity is also low.

The prediction error is large while a scene change occurs and the value of $\mu$ should be larger during scene changes. Therefore, the VSSLMS algorithm can meet our requirements for predicting VBR videos. For practical uses, we adopt the NLMS algorithm instead of the LMS algorithm, so our modified algorithm of the VSSLMS one is called the VSSNLMS algorithm. The weighted vector $W_{n+1}$ update equation of VSSNLMS algorithm is the same as one of the NLMS listed in (3). For an one-step predictor, the value of $x(n+1)$ is not 
available to calculate $e(n)$ at time $n$. Thus, $e(n-1)$ is instead of $e(n)$ and the step-size update equation of the VSSNLMS algorithm should be changed as:

$$
\mu_{n+1}=\alpha \mu_{n}+\gamma e^{2}(n-1)
$$

By the properties of the NLMS algorithm, the VSSNLMS algorithm will converge in the mean if $0<\mu_{\min }<\mu_{\max }<2$. However, in theory of [3], the value $\check{c} g=1$ for the NLMS algorithm provides the fastest convergence, whereas the step size of the NLMS algorithm needs to be considered smaller in practice [4]. By experiments, we set the value $\mu_{\max }=0.5$ in the beginning of prediction. The value of $\mu_{m i n}$ is chosen as 0.1 for smaller misadjustment while there is no scene change occurs.

The value of $\gamma$ is set to $1 \times 10^{-8}$ to detect scene changes properly. Too large value of $\gamma$ causes the value of $\mu$ to be increased even through there is no scene change. On the contrary, too small value of $\gamma$ can not make predictor response to scene changes. The optimal value of is varied by video videos with different autocorrelations. Since the autocorrelation of a real-time VBR video can not be known in prior, it is difficult to determine the optimal value of $\alpha$ before predicting. However, the effect on performance by different values of $\alpha$ is small. By our experiments, the optimal values of $\alpha$ for simulated traces fall in the range from 0.93 to 0.99 . The VSSNLMS predictor chooses $\alpha=0.96$ for simulations.

\section{Analysis and Simulation Results}

This section analyzes the order selecting strategies of the VSSNLMS predictor as well as shows the ability of the VSSNLMS predictor for handling scene changes first. And then we compare the performance between the fixed step-size NLMS predictor and the VSSNLMS predictor by simulations using the MATLAB. The optimal values of step size and the optimal orders using by the fixed step-size NLMS predictor for different VBR videos are listed in Table 1 . These values were obtained by exhausting search and will be used for comparison.

Unlike the order of the NLMS algorithm varied with different autocorrelations of VBR Videos, the VSSNLMS algorithm provided better performance while the order is increased. Figure 1 shows that both the MSE of the "Soccer" and the one of the "Parking Cam" are decreased while the order is increased. Figure 1(a) shows the performance of predicting the "Soccer" is not obviously promoted when the order is increased in excess of 23, whereas Figure 1(b) shows the performance of predicting the "Parking Cam" is not obviously promoted when the order is increased in excess of 6 .

Therefore, there is no confusion for the VSSNLMS to determine the order (i.e. the larger order is, the better performance is). However, the computational 
Table 1. Optimal value of $\mu$ and order for NLMS algorithm

\begin{tabular}{||c||c||c||c||c||c||c||c||}
\hline Trace & Subsequence & $\mu$ & Order & Trace & Subsequence & $\mu$ & Order \\
\hline \hline Jurassic & I & 0.01 & 2 & Soccer & I & 0.07 & 2 \\
Park I & $\mathrm{P}$ & 0.02 & 3 & & $\mathrm{P}$ & 0.03 & 2 \\
& $\mathrm{~B}$ & 0.03 & 3 & & $\mathrm{~B}$ & 0.04 & 2 \\
\hline Silence of & $\mathrm{I}$ & 0.22 & 15 & The & $\mathrm{I}$ & 0.07 & 2 \\
The & $\mathrm{P}$ & 0.06 & 3 & Firm & $\mathrm{P}$ & 0.05 & 12 \\
Lambs & $\mathrm{B}$ & 0.16 & 15 & & $\mathrm{~B}$ & 0.04 & 2 \\
\hline Parking & $\mathrm{I}$ & 0.47 & 11 & Lecture & $\mathrm{I}$ & 0.39 & 10 \\
Cam & $\mathrm{P}$ & 0.21 & 15 & Room & $\mathrm{P}$ & 0.27 & 15 \\
& $\mathrm{~B}$ & 0.19 & 8 & Cam & $\mathrm{B}$ & 0.28 & 15 \\
\hline
\end{tabular}

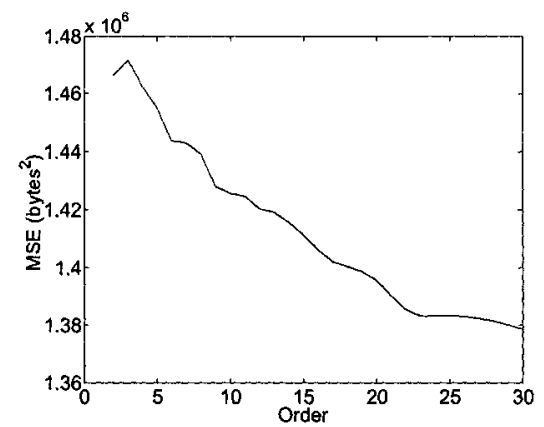

Figure $1(a)$. Performance of the VSSNLMS predictor with increased order (Soccer)

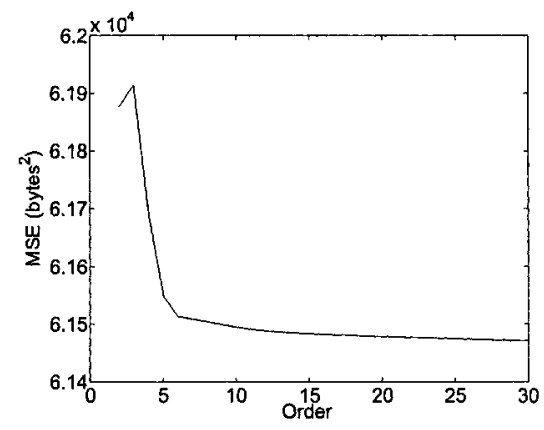

Figure $1(b) . \quad$ Performance of the VSSNLMS predictor with increased order (Parking Cam)

complexity of the VSSNLMS algorithm depends on the order. Too large order causes higher computational complexity of the VSSNLMS algorithm. By experiment, the order $=12$ is good enough for most cases and we adopt order $=$ 12 for comparison.

We compare the ability of handling scene changes between the VSSNLMS algorithm and the NLMS algorithm (with $\mu=0.47$ and $\mu=0.01$ ) for the case of predicting the "Parking Cam". The order used by the NLMS algorithm is 11 and the results is depicted in Figure 2. It shows that the VSSNLMS algorithm has better ability for handling scene changes. And the VSSNLMS algorithm 
can achieve smaller misadjustment if there is no scene change. A scene change occurred from the frame 2030 . The prediction errors of the VSSNLMS algorithm can converge to steady state with the fastest speed. Also, the prediction errors of the VSSNLMS algorithm are smaller than the other two instances in steady state.

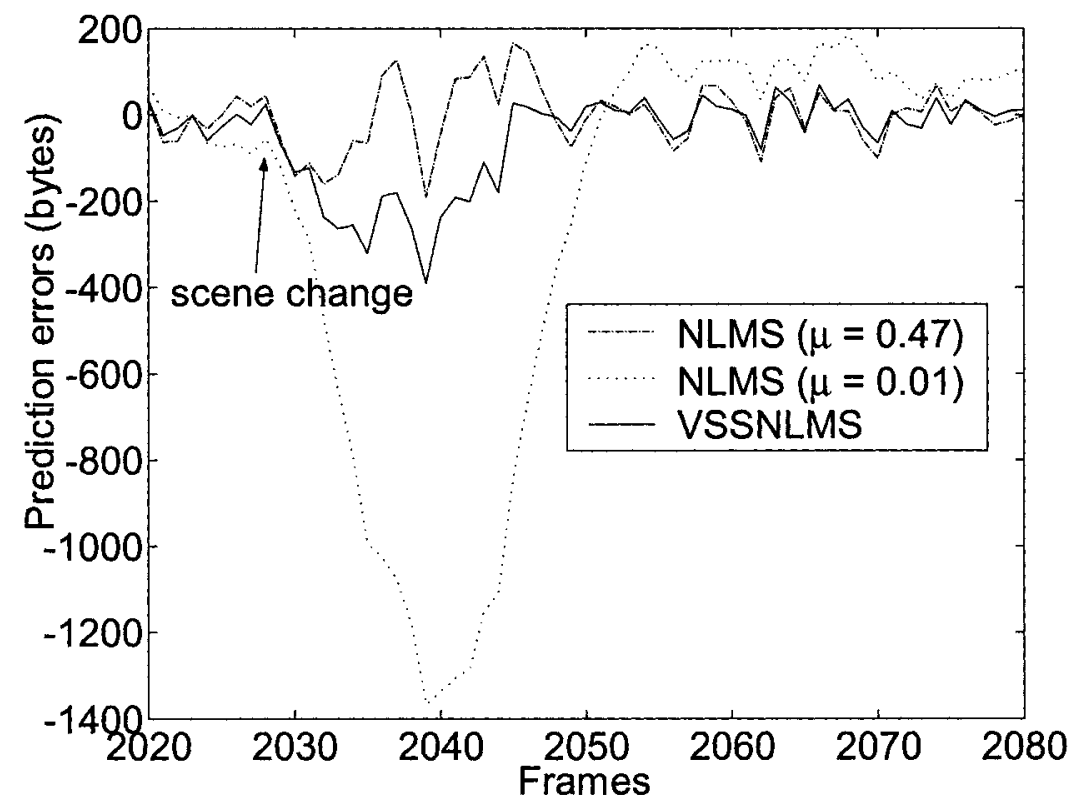

Figure 2. Comparison of the ability for handling scene change

Finally, the comparisons of performance for predicting I-frames, P-frames and B-frames of all MPEG-4 video traces are shown in Table 2. Smaller MSE $\left(b_{t e s}{ }^{2}\right.$ ) indicates better performance and the ratio is defined as that the MSE of the VSSNLMS algorithm divided by the one of the NLMS algorithm. The parameters used by the VSSNLMS algorithm have been discussed in Section 3. The parameters used by the NLMS algorithm are listed in Table 1. The performance of the VSSNLMS predictor is compared with the optimal performance of the NLMS predictor.

The results in Table 2 show that the performances of the VSSNLMS predictor are better or close to the optimal performances of the NLMS predictor. It means that the VSSNLMS algorithm with fixed values of parameters can obtain good performance for most VBR videos with difference statistics and does not need any prior knowledge of VBR videos. Therefore, the VSSNLMS predictor is suitable for the prediction of real-time VBR videos. 
Table 2. Performance comparison for NLMS AND VSSNLMS

\begin{tabular}{||c||c||c||c||c||}
\hline Trace & Subsequence & NLMS (MSE) & VSSNLMS (MSE) & Ratio \\
\hline \hline Jurassic & I & $6.7995 \times 10^{5}$ & $7.4378 \times 10^{5}$ & 1.094 \\
Park I & P & $9.3774 \times 10^{5}$ & $9.5932 \times 10^{5}$ & 1.023 \\
& $\mathrm{~B}$ & $3.1501 \times 10^{5}$ & $3.3754 \times 10^{5}$ & 1.072 \\
\hline Soccer & $\mathrm{I}$ & $1.5527 \times 10^{6}$ & $1.4495 \times 10^{6}$ & 0.934 \\
& $\mathrm{P}$ & $1.3397 \times 10^{6}$ & $1.3179 \times 10^{6}$ & 0.984 \\
& $\mathrm{~B}$ & $3.8252 \times 10^{5}$ & $3.7135 \times 10^{5}$ & 0.971 \\
\hline Silence & $\mathrm{I}$ & $1.2415 \times 10^{6}$ & $1.0353 \times 10^{6}$ & 0.834 \\
of The & $\mathrm{P}$ & $9.9232 \times 10^{5}$ & $8.9586 \times 10^{5}$ & 0.903 \\
Lambs & $\mathrm{B}$ & $4.1607 \times 10^{5}$ & $3.8041 \times 10^{5}$ & 0.914 \\
\hline The & $\mathrm{I}$ & $3.1928 \times 10^{5}$ & $2.9656 \times 10^{5}$ & 0.929 \\
Firm & $\mathrm{P}$ & $4.6159 \times 10^{5}$ & $4.6378 \times 10^{5}$ & 1.005 \\
& $\mathrm{~B}$ & $4.5939 \times 10^{4}$ & $4.3917 \times 10^{4}$ & 0.956 \\
\hline Parking & $\mathrm{I}$ & $6.1875 \times 10^{4}$ & $6.1545 \times 10^{4}$ & 0.995 \\
Cam & $\mathrm{P}$ & $8.4472 \times 10^{4}$ & $1.0586 \times 10^{4}$ & 1.253 \\
& $\mathrm{~B}$ & $7.035 \times 10^{4}$ & $7.9874 \times 10^{6}$ & 1.135 \\
\hline Lecture & $\mathrm{I}$ & $2.5206 \times 10^{4}$ & $2.3031 \times 10^{4}$ & 0.914 \\
Room & $\mathrm{P}$ & $2.4951 \times 10^{4}$ & $2.178 \times 10^{4}$ & 0.873 \\
Cam & $\mathrm{B}$ & $1.5203 \times 10^{4}$ & $1.4246 \times 10^{4}$ & 0.907 \\
\hline
\end{tabular}

\section{Conclusion}

This paper proposed the VSSNLMS predictor which can overcome the problems caused by the fixed step-size LMS-type predictor. The VSSNLMS is based on a variable step-size LMS algorithm which controls step size dynamically by the square of the prediction error. The simulations showed that, without any prior determination of the parameters of the VSSNLMS predictor, the performances of the VSSNLMS predictor are almost better or close to the optimal performances of the fixed step-size LMS-type predictor for the predictions of different VBR videos. Moreover, since the computational complexity of the VSSNLMS predictor is low and the parameters of the VSSNLMS predictor need not be changed for different VBR videos, the VSSNLMS predictor can be implemented in the low-cost IEEE 802.15.3 devices to require predictive bandwidth for different real-time VBR videos.

\section{Acknowledgments}

This work was supported by the MediaTek Inc. under the project "Wireless Communication Systems".

\section{References}


[1] IEEE standard 802.15.3: Wireless Medium Access Control (MAC) and Physical Layer (PHY) Specifications for High Rate Wireless Personal Area Networks (WPANs), Inst. Elec. Electron. Eng., New York, USA, 2003.

[2] A. M. Adas, "Using adaptive linear prediction to support real-time VBR video under RCBR network service model," IEEE/ACM Transactions on Networking, vol. 6, pp. 635$644,1998$.

[3] B.Widrow and S. D. Stearns, Adaptive Signal Processing. Englewood Cliffs, NJ: Prentice-Hall, 1985.

[4] D. P. Mandic, "A generalized normalized gradient descent algorithm," IEEE Signal Processing Letters, vol. 11, pp. 115-118, 2004.

[5] G. Chiruvolu, R. Sankar and N. Ranganathan, "Adaptive VBR video traffic management for higher utilization of ATM networks," it ACM SIGCOMM Computer Communication Review, vol. 28, pp. 27-40, 1998.

[6] J. Foerster, E. Green, S. Somayazulu and D. Leeper, "Ultra-wideband technology for short- or medium-range wireless communications," Journal of Intel Technology, 2nd quarter, 2001.

[7] J. Hosking, "Fractional differencing," it Biometrika, vol. 68, pp. 165-176, 1981.

[8] M. Reisslein et al., "Traffic and Quality Characterization of Scalable Encoded Video: A Large-Scale Trace-Based Study," Arizona State University, Dept. of Elect. Eng., Tech. Rep., Dec. 2003. Video traces available from http://trace.eas.asu.edu.

[9] M. W. Garrett and W. Willinger, "Analysis, modeling and generation of self-similar VBR video traffic," ACM SIGCOMM Computer Communication Review, Proceedings of the conference on Communications architectures, protocols and applications, vol. 24, pp. 269-280, 1994.

[10] N. Sadek, A. Khotanzad, and T. Chen, "ATM dynamic bandwidth allocation using FARIMA prediction model," Proceedings of The 12th International Conference on Computer Communications and Networks, pp. 359-363, 2003.

[11] R. H. Kwong and E. W. Johnston, "A variable step size LMS algorithm," IEEE Transactions on Signal Processing, vol. 40, pp. 1633-1642, 1992.

[12] S. Feng, and R. Sankar, "Limitation of and improvement to linear prediction and smoothing-based bandwidth allocation for VBR traffic," Proceedings of IEEE GLOBECOM '99, vol. 1A, pp. 209-213, 1999.

[13] S. Haykin, Adaptive Filter Theory. Englewood Cliffs, NJ: Prentice-Hall, 1991.

[14] S. J. Yoo, "Efficient traffic prediction scheme for real-time VBR MPEG video transmission over high-speed networks," IEEE Transactions on Broadcasting, vol. 48, pp. 10-18, 2002.

[15] W. E. Leland, M. S. Taqqu, W. Willinger and D. V. Wilson, "On the self-similar nature of Ethernet traffic (extended version)," IEEE/ACM Transactions on Networking, vol. 2, pp. $1-15,1994$.

[16] Y. Shu, Z. Jin, J. Wang and O. W. W. Yang, "Prediction based admission control using FARIMA models," Proceedings of IEEE ICC 2000, vol. 3, pp. 1325-1329, 2000.

[17] Y. Shu, Z. Jin, L. Zhang, L. Wang and O. W. W. Yang, "Traffic prediction using FARIMA models," Proceedings of IEEE ICC'99, vol. 2, pp. 891-895, 1999. 